\title{
Thérèse posthume
}

Posthumous Thérèse of Lisieux

Teresa de Lisieux póstuma

\section{Agnès Desmazières}

\section{OpenEdition}

\section{Journals}

Édition électronique

URL : https://journals.openedition.org/assr/46788

DOI : $10.4000 /$ assr.46788

ISSN : $1777-5825$

Éditeur

Éditions de l'EHESS

Édition imprimée

Date de publication : 5 décembre 2019

Pagination : 161-172

ISBN : 9782713227844

ISSN : 0335-5985

\section{Référence électronique}

Agnès Desmazières, "Thérèse posthume », Archives de sciences sociales des religions [En ligne], 188| octobre-décembre 2019, mis en ligne le 08 janvier 2022, consulté le 08 janvier 2022. URL : http:// journals.openedition.org/assr/46788; DOI : https://doi.org/10.4000/assr.46788 


\title{
Agnès Desmazières
}

\section{Thérèse posthume}

\author{
À propos de: \\ Dauzet Dominique-Marie, Langlois Claude (dir.), Thérèse au tribunal \\ en 1910. Actes du colloque international pour le centenaire $d u$ procès \\ de béatification de Thérèse de l'Enfant-Jésus. Lisieux, 3-5 septembre \\ 2010, Paris, Éditions du Cerf, 2015, 444 p. \\ Guise-Castelnuovo Antoinette, Thérèse de Lisieux et ses miracles. \\ Les recompositions du surnaturel (1898-1928), Paris, Éditions \\ Karthala, coll. «Histoire des mondes chrétiens », 2017, 523 p. \\ LANGLOIs Claude, Les premiers thérésiens: de l'Histoire d'une âme \\ (1898) à la canonisation de Thérèse de l'Enfant-Jésus (1925), Paris, \\ Honoré Champion, coll. "Mystica », 2015, 425 p. \\ LANGLOIs Claude, Thérèse à plusieurs mains. L'entreprise éditoriale \\ de l'Histoire d'une âme (1898-1955), Paris, Honoré Champion, \\ coll. «Mystica ", 2018, 685 p.
}

Depuis vingt ans, le champ de l'histoire de la spiritualité se renouvelle au travers de l'étude de la vie et de l'œuvre de Thérèse de l'Enfant-Jésus, ainsi que de leur réception. Histoire littéraire, histoire de la sainteté, histoire des femmes s'y conjuguent, approfondissant notre étude du catholicisme contemporain. En quoi l'histoire de cette jeune carmélite morte à 24 ans dans la petite ville française de Lisieux peut-elle susciter autant d'intérêt et stimuler notre réflexion? Un dossier des Archives de sciences sociales des religions, intitulé "Theresiana» (2012), dressait un premier bilan à partir des travaux de Claude Langlois sur les manuscrits de Thérèse, où la sainte normande révèle sa vocation d'écrivain.

Dès cette époque, un nouveau champ des études thérésiennes émergeait: l' "après-Thérèse ", la postérité de Thérèse. On le doit au travail pionnier d'Antoinette Guise, mené dans sa thèse remarquable, soutenue en 2006, sur «Thérèse de Lisieux et ses miracles: Recomposition du surnaturel (1898-1928)» (Guise-Castelnuovo, 2017a). Un deuxième jalon de ce nouvel itinéraire est 
signalé par le colloque du centenaire du procès de béatification de Thérèse en 2010 (Dauzet, Langlois, 2015). Suivent deux ouvrages de Claude Langlois sur les "premiers thérésiens ", c'est-à-dire les premiers lecteurs de Thérèse, et sur la rédaction et la réception de l'Histoire d'une âme (Langlois, 2015, 2018).

Plusieurs temporalités se mêlent. Un moment: le tournant de 1910 avec l'ouverture du procès. En 1923, Thérèse est déclarée bienheureuse, le surnaturel est désormais officiellement reconnu. Et, pourtant, est-ce suffisant? N'y faut-il pas une confirmation par la canonisation? Claude Langlois propose ainsi de clore le temps de "premiers thérésiens" par la proclamation de la sainteté de Thérèse en 1925. Antoine Guise-Castelnuovo, quant à elle, poursuit sa recherche jusqu'en 1928, année du vingtième anniversaire de la mort de Thérèse. Celle-ci a été désignée comme patronne des missions l'année précédente. L'étude de l'histoire d'une Histoire d'une âme par Claude Langlois enjambe le procès de béatification et de canonisation pour s'achever, en 1955, au terme de l'aventure éditoriale de l'ouvrage. Quelque cinquante ans du catholicisme français sont ainsi revisités sous le prisme de l'histoire de la réception de la vie et de l'œuvre de Thérèse.

Dans un récent article, Andrea Graus (2019) montre, à partir du cas de trois catholiques françaises (Édith Royer, Thérèse Durnerin et Marthe Robin), comment la mystique épouse de nouvelles formes au tournant du $\mathrm{Xx}^{\mathrm{e}}$ siècle avec l'émergence de profils de femmes mystiques laïques. La sainteté de la vie ordinaire se trouve valorisée. La vocation à la vie mystique est ainsi reconnue comme la vocation de tout baptisé. La considération de l' "après-Thérèse » permet d'élargir le propos en montrant comment la sainte de Lisieux a contribué de manière décisive à la démocratisation contemporaine de la mystique. Toute religieuse qu'elle est, elle en est devenue la figure paradigmatique.

Mon butinage dans ces quatre œuvres s'articulera autour de trois axes principaux: la contribution de l'œuvre thérésienne au renouveau spirituel français; l'hagiographie comme lieu de construction d'une conscience féminine collective; les défis de l'institutionnalisation de la sainteté.

\section{Recompositions du surnaturel}

Comme l'indique bien le sous-titre de l'ouvrage d'Antoinette GuiseCastelnuovo, la réception de la vie et de l'œuvre de Thérèse est symptomatique des « recompositions du surnaturel» qui se font jour au tournant du $\mathrm{Xx}^{\mathrm{e}}$ siècle. Celles-ci ont toutefois de plus lointaines origines que Philippe Boutry retrace dans sa contribution au colloque du centenaire. Il signale ainsi l'avènement d'un «nouveau régime hagiographique (Dauzet, Langlois, 2015:28) autour des années 1840, marqué par un accroissement massif du nombre de béatifications et de canonisations. Une démocratisation de la sainteté s'opère, témoignant d'un nouveau regard sur la sainteté, moins élitiste, plus divers, assumant la variété des situations de vie.

Ce changement de paradigme est dévoilé progressivement. La canonisation de la sainte de Lisieux en représente le point d'orgue. Thérèse de l'Enfant-Jésus 
ne bénéficie pas seulement de ce nouveau régime, mais l'exemplifie par sa vie et par sa doctrine. L'histoire de l'Histoire d'une âme apparaît comme l'histoire d'une prise de conscience "du renouvellement spirituel dont Thérèse est le vecteur et le témoin» (Dauzet, Langlois, 2015: 33). Dans les premières décennies $\mathrm{du} \mathrm{XX}^{\mathrm{e}}$ siècle, à la faveur des rééditions de l'ouvrage et, plus encore, du procès de béatification et de canonisation, croît la conscience que sa vie atteste d'une doctrine qu'elle a elle-même formulée et qui trouve une profonde résonance dans la théologie spirituelle, discipline en pleine renaissance à l'époque.

Antoinette Guise-Castelnuovo y discerne, dans sa conclusion, le «triomphe d'une sainteté normale-exceptionnelle »(Guise-Castelnuovo, 2017a: 479), c'est-à-dire d'une sainteté de la vie ordinaire, qui revêt toutefois des traits exceptionnels justifiant la béatification et la canonisation. C'est bien dans cette tension entre normal et exceptionnel (ou extraordinaire) que se noue la reconfiguration contemporaine du surnaturel. Antoinette Guise-Castelnuovo signale ainsi que "la subversion de la notion de sainteté" est "opérée par Thérèse elle-même» (ibid.: 70). Les péripéties des diverses éditions de l'Histoire d'une âme, ainsi que les vicissitudes du procès de béatification et de canonisation, participent aussi de cette reconfiguration progressive.

Comment accueillir une représentation si paradoxale de la sainteté ? Peut-on porter sur les autels une sainte dont la sainteté est restée cachée de son vivant et dont les écrits font l' "éloge constant de la faiblesse» (ibid.) ? Et, si oui, comment? Tels sont en effet les défis que rencontrent les "premiers thérésiens ». L'histoire des éditions d'une Histoire d'une âme, décrite par Claude Langlois, est l'histoire de son obscurcissement, puis de son dévoilement. Le modèle de sainteté, promu par le carmel de Lisieux au travers de la publication - remaniée, comme le public le découvre progressivement - des écrits autobiographiques de Thérèse, est d'abord celui d'une «sainte artiste, écrivain» (ibid.: 64).

Un tournant se dessine avec la publication, en 1907, de l' «édition" dite «de référence», selon les mots de Claude Langlois (Langlois, 2018: 301). Les profondes révisions apportées au texte sont suscitées par une obscure notice mortuaire de la cousine de Thérèse, qui était elle aussi carmélite sous le nom de Marie de l'Eucharistie. Il est de prime abord surprenant qu'une publication apparemment anodine puisse provoquer des changements aussi importants. En fait, son autrice, mère Agnès de Jésus, sœur aînée de Thérèse et principale artisane de l'Histoire d'une âme, y mentionnait des écrits, jusque-là inconnus, de la future sainte. La "fabrique des paroles" de Thérèse dans l'Histoire d'une âme est ainsi révélée. Se noue ici aussi l' «invention» de la doctrine de l'enfance spirituelle (ibid.: 491). Thérèse n'est plus une simple sainte écrivaine, aux talents littéraires parfois discutés, mais une sainte porteuse d'une doctrine spirituelle que le carmel va s'attacher à divulguer.

Le procès de béatification de Thérèse est aussi le procès de la «sainteté normale-exceptionnelle » qui se déploie dans sa vie et dans ses écrits. Si sa vie est ordinaire, sa doctrine spirituelle ne pourrait-elle pas relever de l'exceptionnel? Le carmel souhaiterait plaider en ce sens. Les clercs aux commandes du procès visent au contraire à en atténuer la portée. En pleine crise moderniste, il est 
dangereux de se recommander d'une «nouvelle» doctrine. En outre, la «petite voie » thérésienne, souvent identifiée à l'abandon, pourrait prêter le flanc à des reproches de quiétisme.

C'est ainsi que prévaut au procès le modèle de la sainte "thaumaturge » (Guise-Castelnuovo, 2017a: 64). Si sa vie reste tout ordinaire ${ }^{1}$, sa postérité, jalonnée de miracles, est baignée de miraculeux. D'où, l'intérêt particulier de la thèse d'Antoinette Guise-Castelnuovo sur les miracles. De quel miraculeux s'agit-il ? À l'ère des conversions fulgurantes - d'intellectuels notamment -, le miraculeux se loge d'abord dans le secret des cœurs, dans les conversions. Il habite également les corps avec les guérisons.

Comme le relève bien Antoinette Guise-Castelnuovo, le miraculeux de Lisieux ne correspond pas exactement à celui de Lourdes. Il ne coïncide pas avec un lieu, mais avec une personne. Il est moins porté par l'expertise médicale que par l'écriture, le récit. La preuve scientifique compte moins que l' "accumulation de l'ex-voto" (ibid.: 343). Promu par le carmel, en particulier au travers de la publication des Pluies de roses, ce "modèle miraculaire" (ibid.: 341) va se confronter à celui promu par la Congrégation des rites, plus proche du modèle lourdais. La béatification ne peut en effet aboutir sans un miracle validé par des médecins.

La tension entre normal et exceptionnel trouve finalement un principe de résolution dans l'affirmation du miracle comme lieu de la confirmation de la sainteté de la vie, ainsi que de l'authenticité de la doctrine (Langlois, 2015 : 186). Claude Langlois l'a bien montré à propos de l'orchestration des témoignages au procès. Le miraculeux de Lisieux n'est donc pas absent du procès, mais relève d'abord de la catégorie du témoignage. Il n'est pas soumis à l'expertise médicale, mais à celle des théologiens, instructeurs du procès.

La «sainteté populaire» (Guise-Castelnuovo, 2017a: 28) de Thérèse ne tiendrait-elle pas justement à cette résolution originale de la tension entre normal et exceptionnel? Claude Langlois décrit ainsi les thérésiens comme "celles et ceux qui la regardent comme un modèle imitable et comme une thaumaturge»(Langlois, 2015: 7). Ordinaire et miraculeux se combinent. Grâce habituelle et grâce actuelle se conjuguent. La dévotion à sainte Thérèse est un lieu de cristallisation de la piété populaire, en particulier autour de la vénération de ses reliques et du pèlerinage à Lisieux. En promouvant par sa "petite voie» la sainteté de la vie ordinaire, Thérèse sort du couvent pour séduire un public de laïcs, hommes et femmes.

Les aspérités de sa vie, en particulier sa nuit spirituelle, centrale pour la compréhension de l'héroïcité de ses vertus et donc de sa sainteté, sont toutefois longtemps occultées (Langlois 2018: 328, 411). La recherche esthétique d'une «belle» sainteté est privilégiée. La beauté du récit est préférée à sa véracité. Craint-on de perdre en popularité ou bien veut-on faire de la beauté un

1. Le miraculeux y apparaît relégué au domaine de l'enfance avec l'épisode de la guérison de la maladie mystérieuse par l'intercession de la Vierge au sourire. 
critère de véracité ? Les doutes et les difficultés de la vie font pourtant partie du quotidien de l'existence. Thérèse rejoint justement dans cette nuit ses contemporains à l'heure de la sécularisation.

Ce silence sur l'épreuve de la nuit peut se comprendre comme une difficulté à penser l'expérience de la nuit spirituelle comme participant de la sainteté de la vie ordinaire. Les thérésiens ne demeureraient-ils pas prisonniers de l'opposition entre l'ascétique de la vie ordinaire et la mystique des expériences spirituelles? Dans son Traité de théologie ascétique et mystique (1938-1939), le dominicain Reginald Garrigou-Lagrange s'en prend d'ailleurs aux auteurs qui jugent que Thérèse, "n'ayant pas reçu de grâces extraordinaires, s'est sanctifiée par la voie ascétique et non par la voie mystique» (Garrigou, 1938: 21). Il témoigne encore combien la lecture de l'Histoire d'une âme a éclairé, trente ans plus tôt, sa propre conception de la nuit de l'esprit (Garrigou, 1929: 578).

Cette difficulté persiste d'ailleurs encore aujourd'hui. La nuit vécue par mère Teresa, qui s'était choisie pour sainte patronne Thérèse de Lisieux, a été ainsi été volontairement cachée à l'issue de son procès. La nuit spirituelle semble relever d'un indicible de la sainteté. Peut-être n'a-t-elle pas été suffisamment considérée comme constitutive de la sainteté. Il serait utile d'étudier ses expressions variées dans les diverses spiritualités. Est-elle l'apanage de la tradition carmélitaine? A-t-elle le même statut dans les autres traditions et comment y est-elle formulée? Comment encore interpréter les tentatives récurrentes de la masquer ? Il serait dès lors important, comme y invite Claude Langlois, de reprendre l'enquête sur la «Thérèse des théologiens» (Langlois, 2018: 527).

\section{Une hagiographie au féminin}

Les trois volumes de Claude Langlois sur les manuscrits de Thérèse, ainsi que son plus récent ouvrage sur la miséricorde chez Thérèse (Langlois, 2016; cf. Guise-Castelnuovo, 2017b), ont révélé combien l'écriture a été le lieu, pour Thérèse, d'une découverte et d'une affirmation de soi. Les quatre ouvrages ici examinés manifestent combien ses écrits ont aussi contribué à faire émerger une conscience féminine collective, en particulier au sein du carmel de Lisieux. Dans Les premiers thérésiens, Claude Langlois fait du couvent normand un véritable "acteur collectif » dont l'identité se forge au travers de la promotion du message thérésien (Langlois, 2015: 55). Comme l'histoire de l'Histoire d'une âme l'atteste, l'écriture de Thérèse ne peut se comprendre qu'au sein d'un milieu, en interaction spécialement avec ses sœurs de sang et ses sœurs de religion, mais aussi avec ses lectrices et lecteurs.

Étudier la postérité de Thérèse invite donc à s'interroger sur la manière dont a pu s'élaborer autour de Thérèse une hagiographie au féminin. Si l'écriture spirituelle féminine a été l'objet d'abondantes études, l'émergence d'une hagiographie au féminin a été moins étudiée. L'on peut d'ailleurs regretter que les quatre ouvrages mentionnés n'assument pas davantage une perspective genrée. Ne peut-on pas penser l'entreprise éditoriale des carmélites comme une entreprise d'affirmation d'un nous collectif féminin? Comment situer 
l'œuvre auto-hagiographique de Thérèse de Lisieux au sein d'une production hagiographique majoritairement masculine? Quelles productions littéraires féminines la vie de Thérèse a-t-elles suscitées ${ }^{2}$ ? Autant de questions qui invitent à une recherche plus ample sur le développement, au $\mathrm{Xx}^{\mathrm{e}}$ siècle, d'une littérature hagiographique produite par des femmes (sur des femmes).

Dominique-Marie Dauzet a souligné, à juste titre, comment le carmel a pu constituer un milieu propice pour l'avènement d'une écriture féminine (Dauzet, 2006: 10). Thérèse est ainsi l'héritière d'une tradition initiée par sa sainte patronne Thérèse d'Avila, tradition qui allie narrativité et poésie, initiative personnelle et réponse à des demandes, comme dans le cas des poésies et pièces de théâtre, divertissements typiquement carmélitains. Il est d'ailleurs remarquable que les carmélites de Lisieux aient insisté pour que l'Histoire d'une âme fasse à ces œuvres de commande, que Thérèse leur avait partagées de son vivant et qui leur étaient destinées, une plus large place que cela était initialement prévu (Langlois, 2018: 156). Si Thérèse s'est découvert progressivement une véritable "vocation d'écrivain »(Goujon, 2009: 441), celle-ci a donc trouvé au carmel un lieu d'épanouissement particulièrement favorable.

Il n'est dès lors pas surprenant que le carmel de Lisieux ait choisi de mettre en avant Thérèse de l'Enfant-Jésus comme sainte "écrivain ». Ce modèle de sainteté peine pourtant à s'exporter hors du carmel. Cela n'empêche pas le carmel de Lisieux de souhaiter franchir une étape supplémentaire en promouvant une sainte "théologienne» (Guise-Castelnuovo, 2017a: 66), élaboratrice d'une nouvelle doctrine. Là aussi, le modèle de la "grande " Thérèse a pu prévaloir. Il est marquant qu'à la même époque émerge l'idée de faire Thérèse d'Avila docteur de l'Église, aux côtés de Jean de la Croix. La singularité de la refondation carmélitaine par une femme et un homme - la femme jouant d'ailleurs à certains égards le rôle de mentor de l'homme, du clerc - n'a-t-elle pas joué en faveur d'une certaine autonomisation du féminin en contexte carmélitain? Une telle tentative d'autonomisation ne peut manquer de se heurter à des résistances. Les réticences des cautions cléricales à mettre en avant au procès la doctrine de Thérèse n'ont sans doute pas tenu seulement à son caractère novateur, mais encore au fait que celle-ci émane d'une femme.

L'attrait pour l'écriture thérésienne - comme, de manière plus générale, celui pour l'expérience mystique féminine - doit beaucoup au fait qu'elle s'élabore «hors de toute surveillance cléricale» (Langlois, 2015: 55). L'histoire d'une Histoire d'une âme manifeste toutefois qu'une caution cléricale est nécessaire lorsque cette écriture franchit la porte du couvent pour se diffuser hors de ses murs. La communauté carmélitaine joue aussi un rôle de médiateur et de régulateur. L'écriture de soi en contexte carmélitain ne peut s'épanouir sans médiations. Cela est déjà vrai du vivant de Thérèse.

2. Je pense notamment aux publications de la tertiaire carmélitaine Jacqueline Vincent, qui ont connu un succès important, en particulier auprès d'un public d'enfants. 
Si Claude Langlois a bien réhabilité la part d'initiative personnelle dans l'écriture thérésienne (Langlois, 2018: 74-78), il n'en demeure pas moins que celle-ci fut portée, du vivant même de Thérèse, par sa prieure, mère Marie de Gonzague, et par ses sœurs. Ces incitations ont d'ailleurs contribué à légitimer les interventions et remaniements dans le manuscrit original thérésien. Etre destinataire ou même commanditaire du texte suffit-il à justifier de tels amendements? Oui, sans nul doute, aux yeux de carmélites qui mènent une vie de dépossession de soi par le vœu d'obéissance.

C'est d'ailleurs un grand mérite de ces travaux de mettre au jour les diverses dynamiques à l'œuvre au sein du carmel de Lisieux, acteur certes «collectif », mais constitué de femmes aux points de vue et stratégies souvent en tension. L'œuvre plurielle de Thérèse nous introduisait déjà dans l'intimité d'un couvent, où vie mystique, travail, divertissement et complexité des relations humaines se mêlaient. L'étude de la postérité de Thérèse nous révèle encore davantage cette complexité.

Le couvent normand présente certes un front commun aux yeux de l'extérieur. Ce qui a sans doute contribué à forger une «légende noire » (GuiseCastelnuovo, 2017a: 22) où mère Agnès de Jésus apparaissait comme la directrice incontestée d'une entreprise de fabrication de la sainteté. La réalité est plus nuancée: mère Agnès a dû négocier avec les membres de sa communauté, comme avec ses appuis cléricaux. Claude Langlois a ainsi fort justement restitué à mère Marie de Gonzague - dont il dresse un portrait plus nuancé que celui que mère Agnès avait cherché à imposer (Langlois, 2018: 63-73) - la paternité de l'idée de publication de l'Histoire d'une âme (ibid.: 137-138).

Dans Thérèse à plusieurs mains, Claude Langlois révèle encore les alliances d'intérêt qui se dessinent au sein de la famille Martin. Mère Agnès de Jésus et sœur Geneviève (Céline) travaillent conjointement - la première par sa plume, la seconde d'abord par ses talents de peintre et de photographe - à l'édification $\mathrm{du}$ «portrait quasi officiel de leur sœur» (Langlois, 2015: 186). Mère Agnès semble avoir même associé sœur Geneviève à son entreprise de réécriture des textes thérésiens (Langlois, 2018: 133). Celle-ci publie d'ailleurs, en 1923, L'esprit de la bienheureuse Thérèse de l'Enfant-Jésus d'après ses écrits et les témoins oculaires de sa vie afin de promouvoir la doctrine spirituelle de sa sœur, qui pâtit de l'attention portée à ses miracles (Langlois, 2018: 470-473).

Marie du Sacré-Cœur, autre sœur de Thérèse et destinataire du manuscrit A, se montre, quant à elle, particulièrement soucieuse de préserver l'authenticité de Thérèse. Elle apparaît davantage comme la caution morale de l'entreprise. Elle est ainsi sollicitée, en 1910, pour «corriger... les corrections » réalisées par mère Agnès au texte de Thérèse (ibid.: 538). Elle entretient aussi des liens particuliers avec la sœur "rebelle», la visitandine Françoise-Marie (Léonie), éditant les lettres que Thérèse lui a adressées (ibid.: 343-344). Léonie, étrangère au carmel de Lisieux, tient un rôle plus effacé, mais son approbation, comme sœur de sang et destinatrice d'écrits, est toutefois recherchée. Elle tient surtout un rôle important au procès en se faisant la promotrice de la doctrine thérésienne de l' «enfance spirituelle» (Langlois, 2015: 378). 
Claude Langlois réhabilite encore la contribution des novices à la diffusion de l'œuvre thérésienne. Il montre bien comment, derrière le collectif du noviciat, se cachent en fait deux novices aux profils très différents, sœur Geneviève et sœur Marie de la Trinité (Langlois, 2018: 373-374), dont le témoignage pèse spécialement au procès (Dauzet, Langlois, 2015 : 395). Leurs paroles font pénétrer au plus intime de la réalité du carmel (Langlois, 2018: 290). Les Conseils et souvenirs des novices montrent aussi Thérèse en dialogue, Thérèse construisant sa pensée au contact de ses novices. Ils témoignent que sa doctrine ne s'est pas élaborée seulement à partir de son expérience spirituelle personnelle ou de sa traversée de la maladie, mais encore au contact de ses sœurs, dans sa fonction de maîtresse des novices. Il serait dès lors intéressant d'examiner ces témoignages des novices dans la perspective d'une histoire de la direction spirituelle au féminin.

Enfin, grâce à ces travaux, deux carmélites «converties de Thérèse », les sœurs Marie-Ange et Isabelle du Sacré-Cœur, sortent de l'ombre. Sœur MarieAnge est l'artisan de la fameuse édition de référence de 1907 et l'instigatrice de l'ouverture de la cause de béatification l'année suivante. Isabelle du Sacré-Cœur oriente le carmel de Lisieux vers une plus grande vulgarisation de l'œuvre de Thérèse. Leur statut de «converties de Thérèse » contribue à légitimer leur intervention dans l'entreprise éditoriale thérésienne. La famille Martin et, au premier chef, mère Agnès, est ainsi conduite à négocier tant avec les anciennes novices qu'avec les converties de Thérèse, tout comme avec la communauté dans son ensemble comme destinataire de certains écrits.

\section{Les défis de l'institutionnalisation de la sainteté}

Avec le procès qui s'ouvre en 1910, le processus de négociation se complexifie, de nouvelles médiations - en particulier cléricales - jouent, et le carmel se trouve pour partie dépossédé de l'héritage thérésien. Comme le note bien François Trémolières, "toute canonisation [...] relève de l'institution et à ce titre, quelle que soit la part de ce qu'il est convenu d'appeler la dévotion populaire dans la "construction" de la figure thérésienne [...], il faut bien qu'il y ait "rencontre", pour ne pas dire convergence, avec le magistère et les préoccupations qu'il formule» (Dauzet, Langlois, 2015: 53).

Antoinette Guise-Castelnuovo - au travers de son étude des miracles, qu'elle conçoit comme une histoire de la "réception de Thérèse par en bas » (Guise-Castelnuovo, 2017a: 30) - s'est attachée à mettre au jour comment la promotion de Thérèse par les carmélites de Lisieux relève d'une «amplification contemporaine de l'adage vox Populi, vox Dei, fondée sur une ecclésiologie implicite, simple et efficace » (ibid.: 196). Philippe Boutry, prolongeant les conclusions d'Antoinette Guise-Castelnuovo, indique que le sensus fidei, « intuition de foi des fidèles et des clercs [...], qui réclament ardemment l'intercession d'une “jeune sainte” qui ne l'est pas encore, même s'il a été assurément alimenté, attisé et structuré par le carmel et les sœurs de Thérèse au premier chef, même si le procès qui fixe les traits de la sainteté "canonique" 
de la Thérèse de 1925 a en partie gommé les aspérités et atténué les vivacités, [...] a été cet élan qui a emporté le cœur des foules, la conviction des clercs et la décision des papes» (Dauzet, Langlois, 2015 : 34).

Claude Langlois l'a bien montré dans Les premiers thérésiens: la popularité de Thérèse déborde les carmels. Le public de l'Histoire d'une âme n'est pas exclusivement féminin: Thérèse convertit des hommes, touche des clercs. Elle reçoit vite une audience internationale grâce à ses miracles. Le succès est populaire et justifie le raccourcissement des délais de la procédure de béatification et de canonisation. Comme le dit bien Antoinette GuiseCastelnuovo, Thérèse est une «sainte qui fait l'unanimité » (Guise-Castelnuovo, 2017a: 228). Il y a donc une singulière convergence autour de Thérèse.

Une telle convergence n'est pas seulement le fruit d'heureuses coïncidences, mais ressort aussi - il est important d'y insister - d'une certaine "fabrique » de la sainteté, comme le met particulièrement en lumière Claude Langlois dans Thérèse à plusieurs mains. Le carmel de Lisieux adapte avec beaucoup d'ingéniosité son offre éditoriale aux différents publics. L'auditoire - à l'exemple des "premiers thérésiens » qui s'expriment eux-mêmes «directement par écrit sur Thérèse » (Langlois, 2015: 8) - ne reçoit pas de manière purement passive l'œuvre thérésienne, mais la lit et l'interprète de façon personnelle, à partir de son propre horizon de préoccupations, à partir de ses propres références spirituelles et intellectuelles. Leur réception de Thérèse influence en retour les productions du carmel. L'Histoire d'une âme est ainsi le résultat d'un processus créatif collectif, faisant dialoguer carmélites, clercs et laïcs.

L'ouverture du procès rend la caution cléricale plus cruciale. Celle-ci apparaissait déjà au principe de l'entreprise éditoriale de l'Histoire d'une âme, comme l'atteste la préface de Godefroid Madelaine, prémontré de l'abbaye de Mondaye, proche de Lisieux. L'ampleur croissante prise, à mesure que la perspective du procès approche, par les lettres d'approbation, émanant de figures cléricales connues, est aussi significative. Le procès contraint le carmel à élargir ses soutiens cléricaux au-delà de son cercle de proches et à confier l'héritage thérésien à des clercs professionnels de la sainteté, tels le vice-postulateur Roger de Teil - expert en procès de canonisation - ou le témoin-clé, Jules Auriault, jésuite, spécialiste de théologie spirituelle. De même, le carmel de Lisieux, tout bérullien qu'il soit, est amené à composer avec les carmes qui ont la responsabilité de la postulation de la cause.

Est-ce justement pour s'émanciper de cette tutelle que mère Agnès, se saisissant de toutes les occasions qui se présentent pour obtenir le soutien des papes successifs, préfère la «logique romaine » à la «logique d'Ordre " (GuiseCastelnuovo, 2017a: 220) ? Une étude de l'intégralité de la correspondance de mère Agnès avec le Saint-Siège contribuerait à éclairer les différents ressorts de son option romaine. Dans une perspective d'histoire des femmes, il serait aussi utile de mettre au jour le statut singulier de mère Agnès comme correspondante des papes. Si, en particulier depuis Jean-Paul II, les amitiés féminines des derniers papes commencent d'être mieux connues, celles de leurs prédécesseurs ont longtemps été un sujet tabou. 
Enfin, et l'historien ne manquera pas de le noter, l'institutionnalisation de la sainteté produit un nouveau rapport à l'histoire. En dépit de la crise moderniste, l'histoire tient une place croissante dans les procédures de béatification et devient, aux côtés de la médecine, une science décisive pour l'authentification de la sainteté ${ }^{3}$. Le procès de Thérèse témoigne de cette évolution. Le débat se cristallise autour de l'authenticité des écrits publiés par le carmel et le procès sur les écrits joue ici un rôle crucial. La demande du tribunal d'effectuer une copie des originaux a, comme le souligne bien Claude Langlois, la «conséquence inestimable de préserver définitivement les originaux de Thérèse » (Langlois, 2018: 541). Si les manuscrits originaux ne sont pas encore accessibles au grand public, la brèche est toutefois ouverte. Les carmes - acteurs obligés du procès - en la personne du premier définiteur général, le Français Marie-Eugène de l'Enfant-Jésus, auront une influence décisive en obtenant de Lisieux l'édition des œuvres complètes, longtemps refusée par mère Agnès.

\section{$* *$ \\ $*$}

L'analyse de ces quatre ouvrages montre la richesse d'une approche croisée de l'histoire de la réception de la vie et de l'œuvre de Thérèse de Lisieux. Réception populaire, "par en bas ", et "fabrique de la sainteté », dont les carmélites sont les actrices - non exclusives -, se rencontrent. Les principaux protagonistes et les grands moments de cette histoire sont ainsi identifiés, les mécanismes de la réception mis en évidence. Comme l'a bien pointé Claude Langlois, il resterait toutefois à faire l'histoire de la réception de l'Histoire d'une âme par les théologiens. Quelle est leur appropriation de l'ouvrage? Quelle place celui-ci tient-il dans leur promotion d'une mystique pour tous? Quel rôle les théologiens ont-ils joué dans l'appropriation populaire de Thérèse de Lisieux? Une enquête approfondie serait utile. Dans cette perspective, une histoire du doctorat de Thérèse serait précieuse.

Nous avons souligné l'importance d'une approche genrée du "cas Thérèse ». Il serait dès lors intéressant de prolonger la réflexion en examinant comment la sainte de Lisieux a pu être présentée comme un prototype de la femme théologienne. Comment l'écriture mystique, en un temps où la théologie spirituelle retrouve ses lettres de noblesse, a-t-elle pu être vecteur d'une entrée des femmes dans le pré carré des hommes et des clercs? Comment des résistances aussi ont-elles pu s'exprimer? Une étude de la trajectoire des «converties » de Thérèse serait importante à cet égard.

Antoinette Guise-Castelnuovo a également mis en lumière, à partir du cas de l'Europe centrale, comment la dévotion à Thérèse s'est progressivement internationalisée, dans le sillage des miracles accomplis (Guise-Castelnuovo,

3. En 1930, Pie XI instituera la «section historique" de la Congrégation des rites, chargée d'enquêter sur les causae antiquae pour lesquelles il n'y a plus de personnes vivantes en mesure de témoigner. 
2017a:184-193). Il conviendrait de reprendre le dossier de façon plus développée sous l'angle d'une histoire globale de la religiosité populaire. Aujourd'hui, les reliques de Thérèse circulent dans le monde entier, suscitant des dévotions largement populaires, y compris dans des pays à majorité non chrétienne. Il serait souhaitable dès lors d'analyser le phénomène sous le prisme d'une histoire de l'inculturation de pratiques de la religiosité populaire. Quels traits prennent ces dévotions? Quels aspects du message de Thérèse sont retenus?

Le regard porté, à travers le cas de Thérèse, sur l'institutionnalisation de la sainteté incite enfin à interroger plus avant les mues du visage de la sainteté, qui s'opèrent au fil de ce processus. Comment enquête historique et investigation théologique se conjuguent-elles, se confrontent-elles aussi parfois, dans une quête commune - qui peut être aussi conflictuelle - de l'«authenticité » de la vie et de la doctrine? Quels critères d'authenticité sont invoqués respectivement par les deux disciplines dans cette «invention» de la sainteté ? Comment l'avènement de la science historique a-t-il bousculé la critériologie théologique?

Agnès Desmazières

Centre Sèvres - Facultés jésuites de Paris agnes.desmazieres@centresevres.com 


\section{Bibliographie}

DAuzet Dominique-Marie, 2006, La mystique bien tempérée. Écriture féminine de l'expérience spirituelle, $\mathrm{XIX}^{e}-\mathrm{XX} \mathrm{X}^{e}$ siècles, Paris, Éditions du Cerf, coll. «Sciences humaines et religions".

Dauzet Dominique-Marie, Langlois Claude (dir.), 2015, Thérèse au tribunal en 1910. Actes $d u$ colloque international pour le centenaire du procès de béatification de Thérèse de l'Enfant-Jésus. Lisieux, 3-5 septembre 2010, Paris, Éditions du Cerf.

Dossier "Theresiana », Archives de sciences sociales des religions, 158, p. 181-247.

Garrigou-Lagrange Reginald, 1938-1939, Traité de théologie ascétique et mystique. Les Trois âges de la vie intérieure [Texte imprimé], prélude de celle du ciel, vol. 1 et 2, Paris, Éditions du Cerf.

Goujon Patrick, 2009, "Les éditions critiques des manuscrits de Thérèse de Lisieux par Claude Langlois. Essai de "poétique spirituelle" ", Recherches de science religieuse, 97, 3, p. 439-445.

Graus Andrea, 2019, “A 'divine mission’ to sanctify the laity: French mystic laywomen and the lay apostolate before Vatican II", Women's History Review, https://doi.org /10.1080/09612025.2019.1590501.

Guise-Castelnuovo Antoinette, 2017a, Thérèse de Lisieux et ses miracles. Les recompositions du surnaturel (1898-1928), Paris, Éditions Karthala, coll. « Histoire des mondes chrétiens".

-, 2017b, compte-rendu de Claude Langlois, Thérèse de Lisieux et la miséricorde, Archives de sciences sociales des religions, 180, p. 378-381.

Langlois Claude, 2015, Les premiers thérésiens: de l'Histoire d'une âme (1898) à la canonisation de Thérèse de l'Enfant-Jésus (1925), Paris, Honoré Champion, coll. "Mystica».

-, 2016, Thérèse de Lisieux et la miséricorde, Paris, Éditions du Cerf, coll. "Spiritualité ».

-, 2018, Thérèse à plusieurs mains. L'entreprise éditoriale de l'Histoire d'une âme (18981955), Paris, Honoré Champion, coll. «Mystica». 